\title{
DISCUSIÓN Y RECOMENDACIONES
}




\section{Discusión}

Acá se presentan algunos resultados del estudio realizado. Estas se dividen en tres partes.

En la primera parte se presenta se presentan resultados y síntesis de partes anteriores. En la segunda, se presenta un argumento sobre la creación de valor en programas de Administración y estudios afines.

\section{Resultados y síntesis de partes anteriores}

Los resultados que se presentan a continuación se enuncian como grandes afirmaciones, seguidas de su argumento, obtenido de la síntesis de las evidencias presentadas en el presente informe, para finalizar cada una con algunas recomendaciones relacionadas con la conclusión enunciada.

1) La formación en Administración parece no alcanzar algunas competencias por menor acceso a medios para mejorar su calidad, y por falta de mayor énfasis en conceptualización y análisis riguroso desde la formación inicial.

Las evidencias indagadas nos muestran que, históricamente, los núcleos básicos de conocimiento de Economía y afines, Humanidades y Medicina obtienen los resultados históricos más altos. También muestra que entre estos núcleos, a nivel histórico, Administración y afines se ubica en los penúltimos lugares, y en los módulos de Lectura Crítica y Comunicación Escrita, en los grupos de puntajes más bajos. Un rasgo que muestran los núcleos de Economía, Humanidades y Medicina es un énfasis en su formación muy fuerte en conceptualización y análisis riguroso.

Otro importante hallazgo en este punto es que los promedios de instituciones, facultades y programas adscritos a ASCOLFA parecen tener mejores puntajes en Saber Pro en Administración y afines, que aquellas que no lo están. Es posible que el trabajo de asociaciones como esta sea un importante instrumento para mejorar la formación que se imparte en los programas de Administración en Colombia.

Recomendación: vincular a ASCOLFA a un número mayor de programas colombianos de Administración y desarrollar, en alianza con ellos y con el Ministerio 
de Educación Nacional, estrategias que lleven a los estudiantes de estos programas a realizar análisis y conceptualizaciones más rigurosos, e incluirlos en planes de mejoramiento y en criterios mínimos de aprobación y acreditación de programas de Administración.

2) La evaluación Saber Pro sigue reflejando mayores desempeños en sus módulos por parte de los estudiantes que habitualmente acceden a recursos y tienen condiciones para avanzar, por lo cual se plantea un gran desafio de formación a estudiantes que no cuentan con estas condiciones.

Los resultados nos mostraron que los altos resultados de algunos de los estudiantes en módulos de competencias genéricas Lectura Crítica, Razonamiento Cuantitativo, Competencias Ciudadanas y, particularmente, Inglés, se relacionan con mayor acceso a recursos materiales, mayores niveles educativos de padres, acceso a instituciones de mayor prestigio, ser hombres, estudiantes en instituciones oficiales, tener internet, tener computador, y contar con menos personas a cargo. La gran duda reside en qué pasa con los estudiantes que dejan de cumplir varias de estas condiciones: estudiantes en programas a distancia tradicional, a distancia virtual, de bajos ingreso o en regiones por fuera de las regiones centrales de Colombia. A pesar de esto, se han hecho importantes avances en los programas con ese otro tipo de estudiantes, como se aborda en la tercera conclusión.

Recomendación: caracterizar detalladamente, a nivel de instituciones y programas, a estudiantes en relación con las variables expuestas en este informe, para complementar los datos de sistemas institucionales de información sobre los estudiantes. Dicha información podría integrarse en un solo sistema unificado a nivel nacional de los programas de Administración, que puede ser administrado por ASCOLFA.

3) Los programas de Administración están aportando importantes capacidades de conocimiento de inglés y de otras competencias genéricas a algunos grupos de estudiantes que habitualmente no son los que presentan más altos desempeños en Saber Pro y en otras evaluaciones externas, en un trabajo donde las evidencias muestran los posibles caminos para el cierre de brechas entre estudiantes de diferentes condiciones y procedencias. 
Un hallazgo de este estudio es que, en muchos casos, los estudiantes en varios programas de Administración son estudiantes de primera generación en sus familias u hogares en acceder a la educación superior, provienen de estratos socioeconómicos que se pueden considerar bajos o medios, en muchos programas no acreditados y en varias instituciones de educación superior no acreditadas. En síntesis: cumplen con características que, como muestra el punto anterior, les pondría en desventaja frente a otros estudiantes.

Los programas de Administración presentan algunos puntos interesantes, precisamente por los logros que muestran con estudiantes en desventaja. El crecimiento en Inglés, de niveles básicos hacia niveles intermedios, teniendo en cuenta que las puntuaciones en Inglés suelen diferenciar mucho más entre los estudiantes con mayores o menores oportunidades en la educación superior, es un punto donde los programas de Administración en Colombia están comenzando a tener una ganancia en aras de cerrar brechas entre estudiantes con más y con menos oportunidades.

En general, los puntajes más altos de Saber Pro del núcleo básico de conocimiento Administración y afines se presentan en Bogotá y Antioquia, y bajos en Cesar, y dentro de los capítulos de ASCOLFA, Bogotá, Antioquia y Suroccidente presentan los puntajes más altos en Saber Pro. A pesar de esto, las tendencias ascendentes en los puntajes en los capítulos Centro y Oriente de ASCOLFA, en los módulos de Lectura Crítica y Razonamiento Cuantitativo, es un punto para fortalecer y promover a estudiantes, por lo cual estas experiencias deben examinarse con detalle.

En concordancia con lo anterior, debe recuperarse la experiencia de trabajo de los departamentos de Colombia que no hacen parte de los departamentos centrales y de mayor dinámica económica, y de las instituciones que logran llevar a los estudiantes de estos departamentos a puntajes altos en modalidades virtuales y a distancia. Igualmente, los puntajes de estudiantes en modalidad virtual están acercándose a los de presencial, y al graduarse parecen tener mayor crecimiento en su vinculación laboral que los estudiantes de otras modalidades (que, de todos modos, ya tienen una tendencia alta), a pesar de la tasa decreciente que muestran los salarios de los graduados de Administración en los últimos años. Todos estos aspectos deben alimentar la política pública y las acciones de mejora de las instituciones y de los programas de Administración en Colombia. 


\section{Recomendaciones}

1). Caracterizar las prácticas que evidencian mayor éxito en llevar al aprendizaje de inglés a estudiantes de programas de Administración, para extenderla al universo de programas colombianos de educación superior en esta área. 2). Caracterizar prácticas de formación en Administración de las instituciones en los capítulos Centro y Oriente de ASCOLFA, para probarlas en otros capítulos de bajos desempeños. 3) Caracterizar y divulgar prácticas de instituciones con alumnos en modalidades distancia virtual y distancia tradicional en los departamentos de Sucre, Meta, Putumayo y Cauca, para probarlas en otros contextos similares e incluir su prueba en sus planes de mejora. 4) Analizar estrategias de las ofertas de formación en Administración a distancia virtual para caracterizar lo que las acerca a las ofertas presenciales, y a partir de dicho análisis generar un plan de todo el conjunto de programas de Administración para diseñar en ellos prácticas en programas no presenciales que exploten lo mejor de la distancia tradicional y la distancia virtual, e incluirlo también como política pública para el fortalecimiento de la formación en esta área.

\section{Creación de valor en programas de Administración y estudios afines}

Un punto importante en este informe es ver el balance de lo presentado y preguntarse si la formación en programas de Administración está creando valor en sus estudiantes, como parte de dicha formación. Es importante destacar que existe una tendencia a que los puntajes más altos de los programas de Administración estén en estudiantes en regiones centrales del país, en el área de sus grandes ciudades, y en estudiantes con mayor nivel educativo de sus padres y con mayores recursos y nivel socioeconómico, que estudian en universidades de mayor prestigio o con admisión selectiva. Si bien el análisis realizado lo insinúa, es importante seguir caracterizando este fenómeno y seguir dando mayor apoyo a la formación en Administración a los estudiantes que no cumplen estas características, para fortalecer más la equidad, en términos de lograr que estudiantes de procedencias más desfavorecidas se ubiquen en los grupos de puntajes más altos en las pruebas (Organización para la Cooperación y el Desarrollo Económico, OCDE, 2016). A pesar de ese panorama, los resultados de este informe nos muestran también algunos puntos de avance con respecto a mayor equidad e inclusión en los programas de educación superior en Administración. 
En primer lugar, los puntajes en pruebas de Saber Pro muestran resultados más altos en metodologías distancia virtual y distancia tradicional, en departamentos fuera de las regiones centrales. Las puntuaciones más altas de estas metodologías, en departamentos como Sucre, Meta, Putumayo o Guainía, muestran que en dichas regiones, con pocas o a veces ninguna institución que ofrezca formación presencial, está llegando a estudiantes en dichas regiones y está logrando que sus estudiantes aprovechen su formación en el área de Administración. Esto es importante, toda vez que las tendencias históricas de puntuaciones de Saber 11, al finalizar la educación básica y media, muestra puntajes más altos de estudiantes en las ciudades de Bogotá y Medellín, y en los Departamentos de Cundinamarca, Boyacá, Santander, Norte de Santander, Antioquia, Caldas, Risaralda y Quindío (Arias-Velandia, Guarnizo-Mosquera, Ortiz-Romero, Gómez-Villareal y Rojas-Benavides, 2018), por lo cual la formación en Administración en distancia tradicional y virtual podría estar dando un valor importante de formación a estudiantes en regiones por fuera de este núcleo. Debe considerarse seriamente, entonces, la experiencia de las instituciones que están impartiendo los programas en estas metodologías, con estudiantes de regiones periféricas de Colombia.

En segundo lugar, es importante destacar lo que sucede con la prueba de Inglés, en la cual los estudiantes del grupo de referencia Administración y afines viene haciendo crecer sus puntajes. La experiencia que tienen en este punto los programas de este grupo es interesante por varios motivos: la diferenciación de grupos de estudiantes que está haciendo la prueba de Inglés (que ya se mencionó en apartados anteriores), mantiene una tendencia, si bien a no estar en los más altos, a ser puntuaciones crecientes en la prueba con el paso de los años, y a lograr que esto se dé en estudiantes de diferentes regiones del país, en especial en regiones donde habitualmente hay puntajes más bajos. En este conjunto, es posible que los programas de Administración estén usando el Inglés, que parece diferenciar estudiantes favorecidos y desfavorecidos, en un área de formación que da a los estudiantes mayores oportunidades para su educación, y como herramienta que puede estar haciendo llegar estudiantes habitualmente desfavorecidos a mayores aprendizajes, con su consecuente mejor ubicación en puntajes de pruebas. Es importante examinar en este punto, con mayor detalle, lo que los programas están haciendo en la formación en Inglés, y cómo podría 
eventualmente convertirse en un "patrimonio de la formación" que se dé por exigencia y demanda a todos los estudiantes del área y que los haga avanzar.

En tercer lugar, es importante mostrar que las regiones con puntajes más altos parecen presentar menores diferencias de puntajes entre sus estudiantes. Este es un punto que dichas regiones pueden explotar para compartir su experiencia con otras, con el fin de enriquecer las oportunidades de los estudiantes de puntos más apartados con una fortaleza ya adquirida por los programas de Administración, que parecen estar cerrando brechas, al menos en regiones centrales del país, entre estudiantes de diferentes condiciones y procedencias.

Por último, las diferencias de puntaje relacionadas con pagos de matrículas nos muestran a veces una tendencia lineal (a mayor pago de matrícula, mayores puntajes en las pruebas de Saber Pro). Sin embargo, es común que, al menos en los primeros grupos de pagos de matrículas, estas tengan una curva en $\mathrm{U}$, donde los puntajes de pagos más bajos o sin pago sean casi iguales a los que empiezan a pagar más de 2 millones 500 mil pesos por matrícula. Esto llama la atención sobre el hecho de que, probablemente, con una mejor inversión de recursos por estudiante y por profesor (como parece pasar en instituciones oficiales, donde pueden estar concentrados varios de estos pagos de matrícula más bajos por estudiante), se estén logrando mejores efectos en el aprendizaje que adquieren los estudiantes. Debe consolidarse una mejor evidencia sobre esta relación, e intentar mejorar las cifras de esta inversión, para comprobar la hipótesis acerca de que mayores puntajes reflejan aprendizaje en función de mayor inversión por profesor y por estudiante en programas de Administración de instituciones colombianas de educación superior. 
REFERENCIAS 
Ahmad, A., \& Maynard, S. (2014). Teaching information security management: Reflections and experiences. Information Management and Computer Security, 22(5), 513-536.

Akben-Selcuk, E., \& Altiok-Yilmaz, A. (2014). Financial literacy among Turkish college students: The role of formal education, learning approaches, and parental teaching. . Psychological Reports, 115(2), 351-371.

Aranda, D., Domínguez, C., Martínez, M., \& Navarro-Paule, A. (2010). Un enfoque innovador del proceso de enseñanza-aprendizaje en la dirección de empresas: El uso de simuladores en el ámbito universitario. Revista de Educación, 353, 707-721.

Arias-Velandia, N., Guarnizo-Mosquera, J., Ortiz-Romero, D., Gómez-Villareal, E., \& Rojas-Benavides, L. (2018). Comienzo de la educación superior virtual en Colombia: entre la concentración geográfica de respuestas institucionales y el cierre de brechas entre zonas rurales y urbanas. En N. Arias-Velandia (Ed.), Aportes a la investigación sobre educación superior virtual desde América Latina. Comunicación, redes, aprendizaje y desarrollo institucional y social. Bogotá D.C., Colombia: Institución Universitaria Politécnico Grancolombiano.

Arias-Velandia, N., Rincón-Báez, W., \& Cruz, J. (2017). Logro educativo en estudiantes de ciencias económicas en educación superior presencial, distancia tradicional y virtual. Informe de investigación presentado por los autores de la Institución Universitaria Politécnico Grancolombiano y Corporación Universitaria Minuto de Dios - Sede Uniminuto Virtual y A Distancia (UVD) al ICFES.

Arismendi-Gómez, F. (2016). La competencia plurilingüe y pluricultural en la formación de futuros docentes de lenguas extranjeras en una universidad pública en Colombia. Folios, 44, 109-125.

Arum, R., \& Roska, J. (201 1). Academically adrift. Limited learning on college campuses. Chicago, IL: University of Chicago Press. 
Banco Mundial. (2009). La calidad de la educación en Colombia: un análisis y algunas opciones para un programa de política. (Vol. 80). Bogotá D.C.: Banco Internacional de Reconstrucción y Fomento/Banco Mundial Misión residente en Colombia.

Busch, D. (2010). Pre-service teacher beliefs about language learning: The second language acquisition course as an agent for change. . Language Teaching Research, 14(3), , 318-337.

Cardona-Román, D., Sánchez-Torres, J., \& Acosta-Márquez, C. (2018). Panorama de la educación virtual en instituciones de educación superior en Colombia. En N. Arias-Velandia (Ed.), Aportes a la investigación sobre educación superior virtual desde América Latina. Comunicación, redes, aprendizaje y desarrollo institucional y social. Bogotá D.C., Colombia: Institución Universitaria Politécnico Grancolombiano.

Carlino, P. (2005). Leer, escribir y aprender en la universidad. Una introducción a la alfabetización académica. Buenos Aires: Fondo de Cultura Económica-Argentina.

Castellanos, Ó. F., Fonseca, S. L., Castrillón, F., Castañeda, L. J., \& Trujillo, G. F. (2013). La Administración del Siglo XXI: Perspectivas para el fortalecimiento de la profesión en Colombia. Bogotá, Colombia: Universidad Nacional de Colombia. Vicerrectoría de Investigación: Consejo Profesional de Administración de Empresas.

Celis, M. T., Jiménez, Ó. A., \& Jaramillo, J. F. (2012). ¿ Cuál es la brecha de la calidad educativa en Colombia en la educación media y en la superior. En ICFES, Estudios sobre la calidad de la educación en Colombia, . Bogotá D.C.: Ministerio de Educación Nacional.

Colombia. Congreso de la Republica. Ley 30 de 1992. (29 de diciembre de 1992). Ley 30 de Diciembre 28 de 1992. Por el cual se organiza el servicio público de la Educación Superior. Diario Oficial 40700. Bogotá D.C., Colombia. Obtenido de http://www.mineducacion.gov.co/1621/ article-86437.html 
Colombia. ICFES. (2 de septiembre de 2011). Resolución 479 de 2011. Por la cual se adoptan las metodologías para seleccionar los mejores estudiantes del Examen de. Bogotá D,C,: Diario Oficial No. 48.180 de 2 de septiembre de 2011 .

Colombia. Ministerio de Educación Nacional. (14 de junio de 2010). Mineducacion. Obtenido de http://www.mineducacion.gov.co/1759/w3-article-231240.html

Colombia. Ministerio de Educación Nacional. Decreto 3963 de 2009. (14 de octubre de 2009). Por el cual se reglamenta el Examen de Estado de Calidad de la Educación Superior. Bogotá D.C. Obtenido de http://www.mineducacion.gov.co/1621/articles-205955_archivo_pdf_decreto3963.pdf

Engelhard, G. (1994). Examining rater errors in the assessment of writing composition with a many-faceted Rasch model. Journal of Educational Measurement, 3 I (I), 93-1 12. doi:http://dx.doi.org/10.1 1 l 1/j.1745-3984.1994. tb00436.x

Guarín, A., Londoño, S., Medina, C., Parra, J., Posso, C., \& Vélez, C. E. (2016). Estimating the Effect of Attending a Public versus a Private University in Colombia on Academic Achievement. Borradores de Economía (968), 1-50.

Gutiérrez-Rodríguez, M., \& Flórez-Romero, R. (2012). Enseñar a escribir en la universidad: saberes y practicas de docentes y estudiantes universitarios. . Magis, Revista Internacional de Investigación en Educación, 4(7),, 137-168.

Hederich-Martínez, C. (2007). Estilo cognitivo en la dimensión de dependencia-independencia de campo. Influencias culturales e implicaciones para la educación. . Bogotá D.C.: Universidad Pedagógica Nacional.

Hederich-Martínez, C., \& Camargo-Uribe, A. (2000). Estilo cognitivo y logro educativo en la ciudad de Bogotá D.C. Bogotá D.C.: Centro de Investigaciones de la Universidad Pedagógica Nacional (CIUP) / Instituto para la Investigación Educativa y el Desarrollo Pedagógico (IDEP), Alcaldía Mayor de Bogotá D.C. 
ICFES. (2016a). Guía de orientación: Modulo de Lectura Critica Saber Pro 2016-2. Instituto Colombiano para la Evaluación de la Educación . Bogotá D.C.: ICFES. Recuperado el 05 de octubre de 2017, de http://www.icfes.gov.co/docman/estudiantes-y-padres-de-familia/ saber-pro-estudiantes-y-padres/estructura-general-del-examen/modulos-saber-pro-2016-2/modulos-primera-sesion-competencias-genericas-12/2336-guia-de-orientacion-modulo-de-lectura-critica-saber-

ICFES. (2016b). Guía de orientación. Módulo de Razonamiento Cuantitativo, Saber Pro 2016-2. Bogotá D.C., Colombia: Instituto Colombiano para la Evaluación de la Educación, ICFES.

ICFES. (2016c). Guía de orientación. Módulo de Competencias Ciudadanas, Saber Pro 2016-2. Bogotá D.C., Colombia: Instituto Colombiano para la Evaluación de la Educación, ICFES.

ICFES. (2016d). Guía de orientación. Módulo de Comunicación Escrita, Saber Pro 2016-2. Bogotá D.C., Colombia: Instituto Colombiano para la Evaluación de la Educación, ICFES.

ICFES. (2016f). Guía de orientación. Módulo de Gestión Financiera, Saber Pro 20162. Bogotá D.C., Colombia: Instituto Colombiano para la Evaluación de la Educación, ICFES.

ICFES. (2016g). Guía de orientación. Módulo de Gestión de Organizaciones, Saber Pro 2016-2. Bogotá D.C., Colombia: Instituto Colombiano para la Evaluación de la Educación, ICFES.

ICFES. (2017). Descripción bases de datos. Bogotá D.C., Colombia: Instituto Colombiano para la Evaluación de la Educación. Recuperado el 10 de mayo de 2017, de http://www.icfes.gov.co/investigadores-y-estudiantes-posgrado/descripcion-bases-de-datos

ICFES. (2017a). Guía de Orientación SABER PRO, Módulos de competencias Genéricas. Bogotá D.C.: Instituto Colombiano para la Evaluación de la Educación (Icfes). 
ICFES. (2017c). Guía de Orientación. Saber Pro, Competencias Específicas. Formulación, evaluación y gestión de proyectos. Bogotá D.C.: Instituto Colombiano para la Evaluación de la Educación (Icfes).

ICFES. (2016e). Guía de orientación. Módulo de Inglés, Saber Pro 2016-2. Bogotá D.C., Colombia: Instituto Colombiano para la Evaluación de la Educación, ICFES.

Ministerio de Educación Nacional de Colombia. (2007). Glosario de la Educación Superior. Recuperado el 26 de 06 de 2017, de Ministerio de Educación Nacional: https:/www.mineducacion.gov.co/sistemasdeinformacion/1735/articles-213912_glosario.pdf

Ministerio de Educación Nacional de Colombia. (2016). Documento metodológico del Modelo de Indicadores del Desempeño de la Educación (MIDE). (M. d. Colombia, Ed.) Recuperado el 06 de 11 de 2017, de Colombia Aprende: La red del conocimiento: http://52.37.84.217/edu-mide/documents/Documento\%20metodol\%C3\%B3gico\%20MIDE\%202.0\%20\%20VF.pdf

Ministerio de Educación Nacional, Observatorio Laboral para la Educación. (10 de 12 de 2017). Observatorio Laboral para la Educación OLE. Obtenido de http://www.graduadoscolombia.edu.co/html/1732/propertyvalue-36299.html

Ministerio de Educación Nacional. CNA. (2013). Lineamiento para la acreditación de programas de pregrado. Bogotá D.C.: CNA. Obtenido de https:// www.cna.gov.co/1741/article-186377.html

Ministerio de Educación Nacional-CNA. (2017). CIFRAS DEL SISTEMA NACIONAL DE ACREDITACIÓN A 3I DE DICIEMBRE DE 2016. Bogotá D.C.: Consejo Nacional de Acreditación. Obtenido de https:// www.cna.gov.co/1741/articles-322100_Boletin_2016_def.pdf

Organización para la Cooperación y el Desarrollo Económico, OCDE. (2016). Resultados de PISA 2015. París, Francia: Organización para la Cooperación y el Desarrollo Económicos, OCDE. 
Ramírez, C. (2014). Factores asociados al desempeño académico según nivel de formación pregrado y género de los estudiantes de educación superior Colombia. Revista Colombiana de Educación, (66),, 203-224. Recuperado el 06 de 12 de 2017, de http://www.scielo.org.co/pdf/rcde/n66/n66a09.pdf

Rodríguez, A. G., Gómez, L. V., \& Ariza, D. M. (2014). Calidad en educación superior a distancia y virtual: un análisis de desempeño académico en Colombia. Investigación y Desarrollo, 22(1), 58-99. 